\title{
Seroprevalencia de marcadores infecciosos causantes de pérdidas de hemodonaciones en el Servicio de Banco de Sangre del Hospital Nacional Docente Madre Niño San Bartolomé de enero 2008 a diciembre del 2013
}

\author{
Jeél Moya $\mathrm{S}^{1}$, Edward Julcamanyan $\mathrm{T}^{2}$
}

\begin{abstract}
RESUMEN
Objetivo: Determinar la seroprevalencia de marcadores infecciosos causantes de pérdidas de hemodonaciones en el Servicio de Banco de Sangre del Hospital Nacional Docente Madre-Niño San Bartolomé de enero 2008 a diciembre del 2013.

Material y Métodos: Estudio retrospectivo, de corte transversal, descriptivo. El criterio de inclusión fue hemodonaciones completas sin complicaciones que cumplían con los criterios de calidad y normatividad del PRONAHEBAS. El análisis de datos se realizó en tres procesos básicos: codificación, tabulación y construcción de tablas y gráficos. La técnica utilizada para la verificación estadística de los resultados fue mediante el analizador estadístico SPSS versión 20.0.

Resultados: Los hallazgos fueron: $4.63 \%$ para HBCAb, 1.78\% para sífilis, 1.21\% para HTLV I-II, y 5.31\% para otros marcadores serológicos de un total de 11399 donaciones completas. La prevalencia general fue de $9.36 \%$ para todos los marcadores, lo cual ocasiono una pérdida de 1016 donaciones; 457.2 Litros de sangre y 61,893.28 USD perdidos. Las asociaciones entre marcadores infecciosos más frecuentes fueron: HBcAb con sífilis y HBsAg y los tres componentes que explican la varianza fueron asociados por cronicidad y epidemias concentradas en poblaciones, por exposición ocupacional y por relación subrogante.

Conclusión: La prevalencia hallada demostró la mala calidad de donantes de sangre y el gran impacto económico por hemoderivados desechados muestran las limitaciones en la cadena de donación. Por lo que es conveniente continuar con las campañas de educación sanitaria, las buenas prácticas en medicina transfusional y la selección de donantes de sangre para prevenir las infecciones transmisibles por transfusión, aumentar el suministro de sangre sin poner en riesgo al receptor y sin nuevas donaciones, asimismo reducir el costo económico perdido por donación. (Horiz Med 2014; 14(4): 6-14)
\end{abstract}

Palabras clave: seroprevalencia, hemodonante, marcadores infecciosos, tamizaje. (Fuente: DeCS BIREME).

Seroprevalence Infectious Markers Causing Loss of Donations in the Blood Bank Service in Hospital Nacional Docente Madre - Niño San Bartolomé January 2008 to December 2013

\begin{abstract}
Objective: To determine the seroprevalence of infectious markers causing loss of blood donations in the Blood Bank Service in Hospital Nacional Docente Madre Niño San Bartolome January 2008 to December 2013.

Material and Methods: A retrospective, cross-sectional, descriptive study was conducted. Inclusion criteria were donations of whole blood without complications that meet the quality criteria and standards of PRONAHEBAS. Data analysis was conducted in three basic processes: coding, tabulation and construction of tables and graphs. The technique used for the statistical verification of the results was by SPSS version 20.0 statistical analyzer.

Results: The findings were $4.63 \%$ for HBCAb, $1.78 \%$ for syphilis, $1.21 \%$ for antibodies to HTLV I-II, and $5.31 \%$ for other serological markers of a total of 11399 complete donations. The overall prevalence was $9.36 \%$ for all markers which caused a loss of 1016 donations, 457.2 liters of blood, and 61893.28 USD lost. Similarly, frequent associations between infectious markers were $\mathrm{HBCAb}$ that of syphilis and $\mathrm{HBsAg}$, and the three components explaining the variance were associated with chronicity and concentrated epidemics in populations, occupational exposure and surrogate relationship.

Conclusion: The prevalence found shows the poor quality of blood donors and the high economic impact of discarded blood show the limitations of the donation chain. So it is appropriate to continue sanitary education campaigns, best practices in transfusion medicine and selection of blood donors to prevent transfusion-transmissible infections, increase the blood supply without compromising the receiver without new donations also reduce the economic cost lost by donation. (Horiz Med 2014; 14(4): 6-14)
\end{abstract}

Key words: seroprevalence, blood donor, infectious markers, screening. (Source: MeSH NLM).

Tecnólogo médico en Laboratorio clínico y anatomía patológica. Universidad Privada Norbert Wiener Lima - Perú.

Lic. TM. Servicio de Banco de Sangre y Hemoterapia Tipo II. Hospital Nacional Docente Madre Niño San Bartolomé Lima - Perú. 
Seroprevalencia de marcadores infecciosos causantes de pérdidas de hemodonaciones en el Servicio de Banco de Sangre del Hospital Nacional Docente Madre Niño San Bartolomé de enero 2008 a diciembre del 2013

\section{INTRODUCCIÓN}

La transfusión sanguínea, representa un potencial vehículo de propagación de enfermedades infecciosas. El riesgo por transfusión en los Estados Unidos de América, se estima desde 1 por cada 4,405 donaciones para T. pallidum hasta 1 por cada 79,986 donaciones para Trypanosoma cruzi (1).

En países primermundistas, las infecciones bacterianas exceden a los agentes virales, la incidencia de reacciones transfusionales por bacterias es entre 1 por cada 100.000 unidades en el caso de los concentrados de glóbulos roj os, y entre el 1 por 900 unidades en el caso de los concentrados de plaquetas (2). Con un riesgo de infección de plaquetas mayor que el paquete globular (3).

En países en desarrollo, el riesgo relativo por donación es heterogéneo, debido a la diversidad geográfica, el hábitat y los grupos de población. La probabilidad de infecciones varían entre 0.95 por 10 mil donaciones para el Virus de la Inmunodeficiencia Humana (VIH), 20.0 - 30.0\% para Hepatitis B y C y el riesgo más eminente es para la enfermedad de Chagas, sobretodo en Perú y Bolivia $(4,5)$.

El aseguramiento de sangre en su totalidad aún no se instaura, sobre todo en países con políticas de salud y prácticas laboratoriales deficientes.

El procesamiento de las hemodonaciones requiere como mínimo: determinación del grupo $A B O$, el factor Rh0 (D), el reconocimiento de anticuerpos irregulares y el tamizaj e de marcadores infecciosos: antígeno superficial (AgHBs) y antígeno core del virus de la hepatitis B (anti-HBC), anticuerpos contra VIH tipo I o II (anti-VIH 1 y anti-VIH 2), anticuerpos contra el virus de la hepatitis C (antiVHC) y serología de sífilis (6). Anticuerpos contra los virus linfotrópicos humanos (anti-HTLV I-II), y marcadores para la enfermedad de Chagas, en zonas endémicas (7).

Las infecciones transmisibles por transfusión (ITT), son efectos adversos tardíos que aumenta el riesgo en hemodonaciones. Principalmente, la infección por hepatitis B y C, el VIH/SIDA, la sífilis y las secuelas ocasionadas por los HTLV y Chagas (8-13).

En américa latina, se consideran dos vías de transmisión: la actividad sexual no segura y la intrafamiliar infantil (amazonía) (14). Esto genera 2 millones de personas infectadas a nivel mundial y 350 millones con infección crónica por el VHB, con incremento de seropositividad en mayores de 60 años $(15,16)$. Situación parecida a la de prevalencia chagásica, causa número dos de ITT en países endémicos y que produce 10 millones de infectados principalmente en Sudamérica (17).

El riesgo de transfusión de Trypanosoma cruzi se incrementa en proporción a la prevalencia de la infección en los donantes de sangre (18). Esta endemia global, en el Perú, se desarrolla con regularidad con riesgo promedio en transfusiones alogénicas (79.98\%) (19).

Las conductas de riesgo, la calidad de donantes y las endemias geográficas generan una inconstancia de factores que dificultan la labor de los bancos de sangre, donde el tamizaje de marcadores infecciosos constituye la medida invariable para eliminar sangre insegura, tal y como lo establece la Organización Panamericana de la Salud (OPS) (20).

Otras enfermedades potencialmente transmisibles por sangre o sus derivados, son: dengue, enfermedad de Lyme, Malaria, Citomegalovirus (CMV), enfermedad de Crutzfeldt-Jakob y las infecciones por el virus del Ebola y Marburg, entre otras; de las que existen reportes aislados pero significativos (21-27).

El tamizaje de marcadores anti-CMV es útil para prevenir su transmisión $(28,29)$, importante en la población materna infantil.

Las donaciones voluntarias no remuneradas en nuestro país, mantienen un porcentaje menor del $25 \%$ del total de donaciones por año (30).

Diversos estudios en Perú, señalan una prevalencia de 1 a $2.2 \%$ para donantes portadores del $\mathrm{HBsAg}$, 11.6 casos de portadores con VIH por 1000 donantes y la frecuencia de resultados positivos para VDRL varían entre $0.66-4.1 \%$. Esto incrementa el riesgo relativo por donación y confirma la poca calidad de hemodonaciones (31-33).

La variación en el costo refleja principalmente las diferencias en la prevalencia y el precio de prueba establecido por cada Centro de Salud, pero además; las unidades seropositivas reflejan la cantidad de 
sangre perdida por los bancos de sangre (34-36). Lo cual genera un gran impacto económico hacia la institución.

La autoexclusión, no parece ser un medio de control y la hemovigilancia asumiría más intervención con el uso de nuevas tecnologías de tamiz (37-39).

El objetivo de la presente investigación, fue determinar la seroprevalencia de marcadores infecciosos causantes de pérdidas de hemodonaciones.

\section{MATERIAL Y MÉTODOS}

Estudio retrospectivo, de corte transversal, descriptivo.

Población: Todo hemodonador (donante voluntario y por reposición) del Servicio de Banco de Sangre, del HONADOMANI SB, de Enero del 2008 a diciembre del 2013.

Muestra: Se extrajo la muestra respetando los criterios establecidos previamente.

Criterios de inclusión: hemodonaciones completas sin complicaciones que cumplan con los criterios de calidad y normatividad del Programa Nacional de Hemoterapia y Bancos de Sangre (PRONAHEBAS), y que al tamizaje resulten seropositivos a partir de un marcador infeccioso.

Edad de los hemodonadores: entre 18 y 55 años.

Criterios deexclusión: hemodonacionesincompletas, frustras o no reactivas al tamizaje. Asimismo, las colectadas sin completar los requerimientos mínimos del Servicio o evidentemente contaminadas.

Técnica de recolección de datos y procesamiento de la muestra: Se realizó por medio del Registro de Donaciones del Servicio, discriminado por años, sexo, complicaciones y reactividad a marcadores serológicos. Los datos considerados dentro de la investigación fueron los que resultaron reactivos a más de un marcador infeccioso, unidades que fueron desechadas luego del cribado obligatorio establecido por Ministerio de Salud-PRONAHEBAS. El tamizaje se realizó por inmunoensayo enzimático (biokit USA inc. y Bio-Rad, inc.). Los marcadores infecciosos considerados dentro del tamizaje fueron AgHBs, anti-HBc, anti-VIH 1/anti-VIH 2, anti-VHC, anti-HTLV I-II, CHAGAS y serología de sífilis. Todos los procesos siguieron un Procedimiento Operacional Estandarizado y cumplieron con los controles de calidad según el PRONAHEBAS.

Técnica de Análisis de Datos: Se realizó en tres procesos básicos: codificación, tabulación y construcción de tablas y gráficos. La técnica utilizada para la verificación estadística fue mediante el analizador estadístico SPSS versión 20.0 y Microsoft Excel para Windows. La correlación de variables se realizó mediante la prueba de esfericidad de Barlett y KMO, resultando la matriz de correlaciones entre variables adecuada $(p=<0.05)$. Conjuntamente, se realizó el Análisis factorial por Rotación Varimax.

Ética: la información obtenida se mantendrá en anonimato y sólo se utilizará para esta investigación.

\section{RESULTADOS}

Los resultados pueden extrapolarse a otros bancos de sangre, ya que los donantes cumplieron con los criterios de selección y calidad estandarizados por el PRONAHEBAS, lo que implicó que los donantes compartieran características básicas.

De las 11,399 unidades de sangre, se observó un aumento promedio de $15 \%$ de donaciones por año. Esto principalmente por donaciones voluntariarelacionadas, por reposición y depósito.

La prevalencia de hepatitis B se mantuvo constante (4.63\%). Tabla 1y figura 1.

La prevalencia de VIH en los donantes de sangre ha disminuido de manera constante, $0.51 \%$ para el 2005-2010 a 0.41\% para el 2013. Tabla 2 y 3.

El descarte de unidades de sangre a causa de marcadores positivos aumentó de $0.82 \%$ el 2010 a $1.54 \%$ el 2011. Tabla 4 y figura 2. 
Seroprevalencia de marcadores infecciosos causantes de pérdidas de hemodonaciones en el Servicio de Banco de Sangre del Hospital Nacional Docente Madre Niño San Bartolomé de enero 2008 a diciembre del 2013

Tabla 1. Total de muestras seropositivas para 07 marcadores infecciosos durante el periodo de estudio en 11,399 donaciones completas.

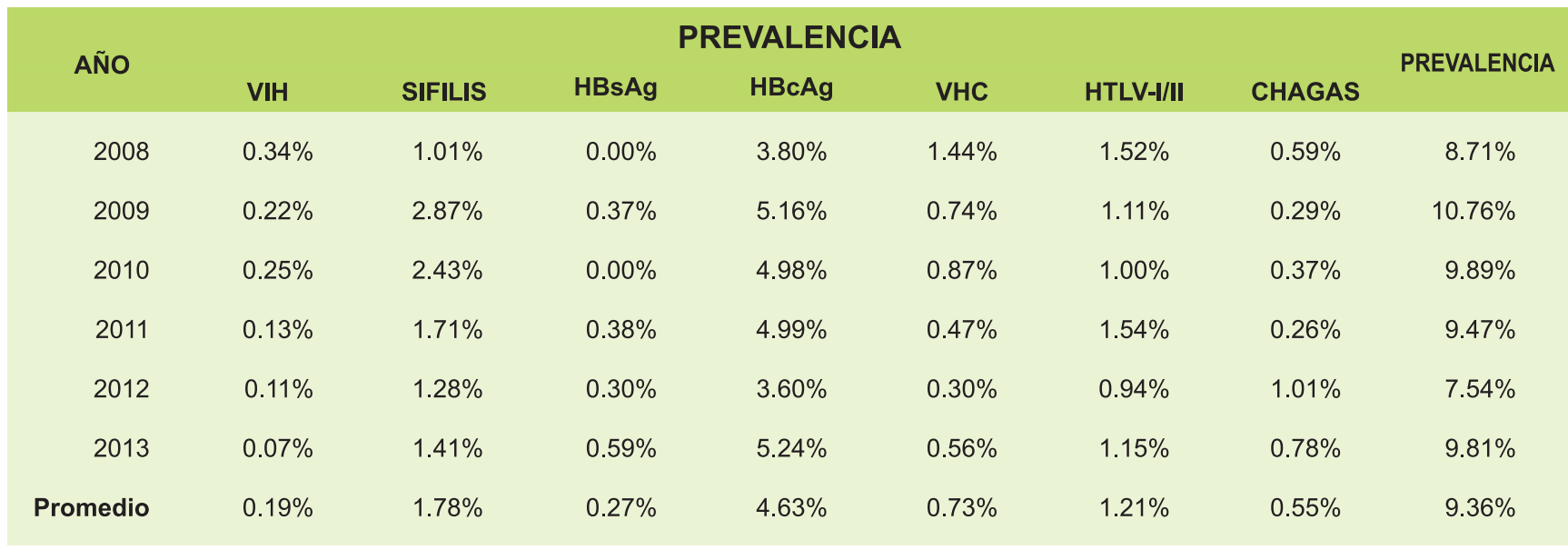

Figura 1. Prevalencia de marcadores infecciosos 2008-2013.

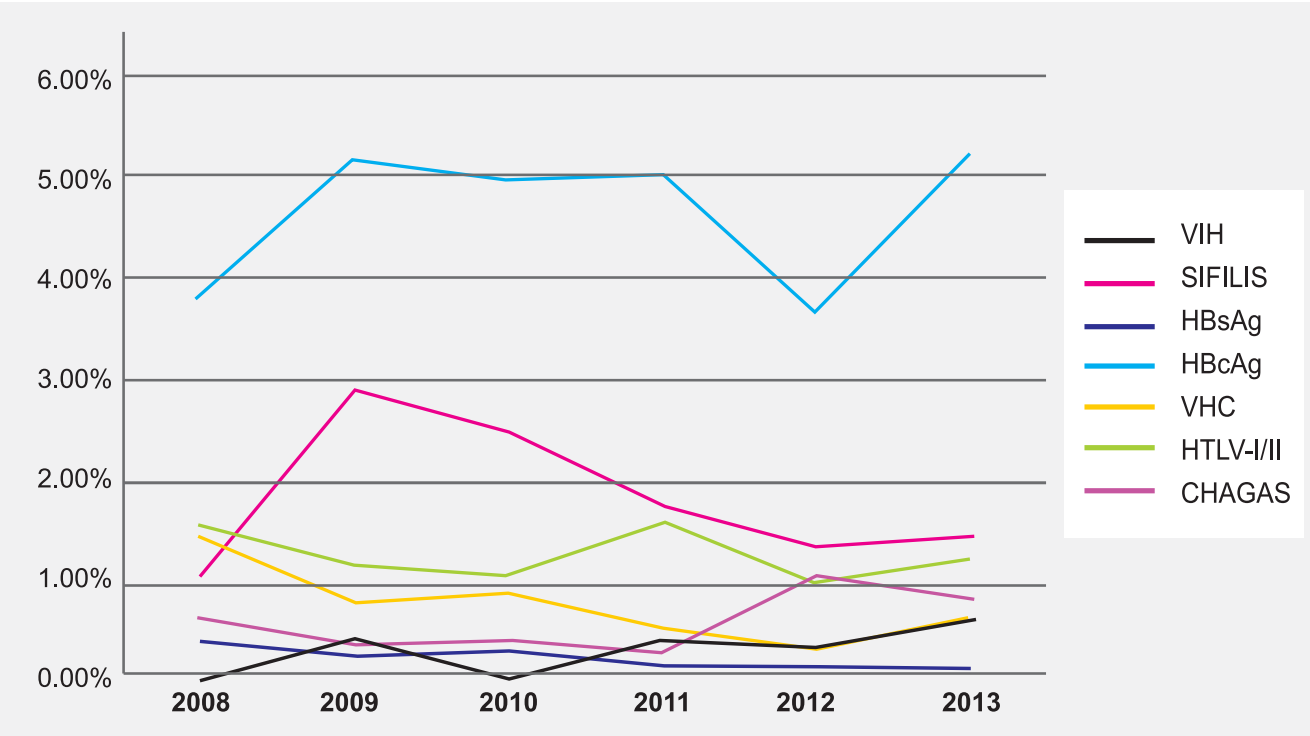

Tabla 2. Pérdida de sangre y costo, discriminados por años, considerando un volumen aproximado de $450 \mathrm{~mL}$ de sangre por donación.

\begin{tabular}{|c|c|c|c|c|c|c|c|}
\hline AÑOS & 2008 & 2009 & 2010 & 2011 & 2012 & 2013 & TOTAL \\
\hline TOTAL SEROPOSITIVOS & 103 & 146 & 159 & 222 & 201 & 264 & 1095 \\
\hline PERDIDA DE SANGRE (litros) & 41,75 & 60,75 & 66,6 & 91,8 & 85,05 & 110,25 & 457,2 \\
\hline
\end{tabular}

* Unidades eliminadas por año, considerando 2 - 3 marcadores infecciosos positivos para un mismo hemodonador

** Considerando el precio por bolsas TERUMO y el estudio del completo del hemodonador. Costo unitario: 170 nuevos soles. 


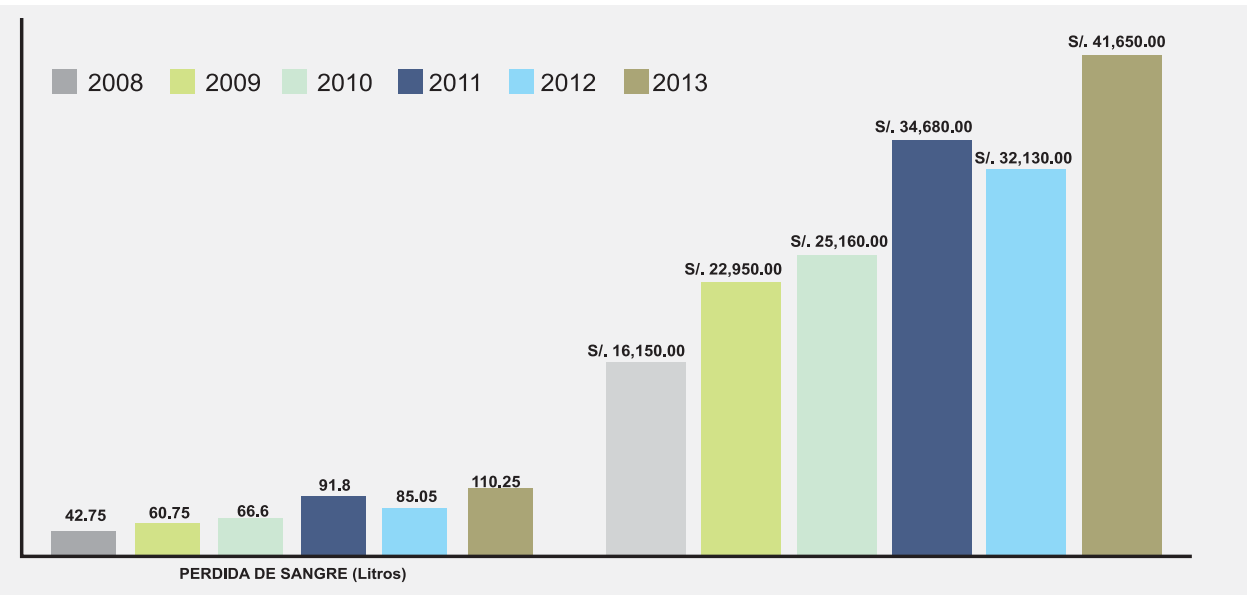

Figura 2. Proporción de unidades de sangre y costo perdido por hemodonaciones seropositivas, 2008-2013.

Tabla 3. Asociaciones entre marcadores infecciosos seropositivos durante 2008-2013.

$\begin{array}{cccccccc}\text { Asociaciones // Años } & 2008 & 2009 & 2010 & 2011 & 2012 & 2013 & \text { Total } \\ 2 \text { marcadores } & & & & & & & \\ \text { VHI + HBcAb } & 1 & 0 & 0 & 0 & 0 & 0 & 1 \\ \text { SIFILIS + HBcAb } & 1 & 3 & 7 & 4 & 3 & 4 & 22 \\ \text { HBsAg+HBcAb } & 0 & 4 & 0 & 7 & 4 & 9 & 24 \\ \text { VCH+HTLV I/II } & 1 & 0 & 0 & 0 & 0 & 1 & 2 \\ \text { VHI + HBcAb } & 0 & 0 & 0 & 0 & 0 & 0 & 0 \\ \text { CHAGAS + HBcAb } & 0 & 0 & 1 & 0 & 3 & 1 & 5 \\ \text { HTLV I/II + HBcAb } & 0 & 0 & 1 & 6 & 1 & 1 & 9 \\ \text { VHI + SIFILIS } & 0 & 0 & 0 & 0 & 1 & 1 & 2 \\ \text { VHC + HBcAb } & 3 & 2 & 1 & 0 & 0 & 0 & 6 \\ \text { VHC + CHAGAS } & 1 & 0 & 0 & 0 & 0 & 0 & 1 \\ \text { VIH + HTLV I/II } & 0 & 0 & 1 & 0 & 0 & 0 & 1 \\ \text { SIFILIS + HTLV I/II } & 0 & 2 & 0 & 0 & 0 & 0 & 2 \\ \text { 3 marcadores } & & & & & & & \\ \text { VHC + HTLV + CHAGAS } & 1 & 0 & 0 & 0 & 0 & 0 & 1 \\ \text { HBsAg + HBcAb + CHAGAS } & 0 & 0 & 0 & 1 & 0 & 0 & 1 \\ \text { SIFILIS + HBsAg + HBcAb } & 0 & 0 & 0 & 0 & 0 & 2 & 2 \\ \text { Total } & 8 & 1 & 11 & 18 & 12 & 19 & 79\end{array}$

Tabla 4. Elementos que explican la varianza de todas las pruebas. Nótese los tres componentes estadísticos extraídos y asociadas por factores comunes.

\begin{tabular}{llll}
\multicolumn{5}{c}{$\begin{array}{c}\text { Matriz de componentes } \\
\text { Componente } \\
\end{array}{ }^{\mathrm{a}}$} & \multicolumn{3}{c}{3} \\
HBcAg &, 813 &, 229 & \\
HBsAg &, 763 &,- 129 &, 455 \\
CHAGAS &, 591 &,- 377 &, 210 \\
HTLV - I/II &, 525 &, 362 & \\
VHC & &, 879 &,- 623 \\
SIFILIS &, 459 &, 147 &, 594 \\
VIH &,- 196 &, 196 &
\end{tabular}

Método de extracción: Análisis de componentes principales

a. 3 componentes extraídos

\section{DISCUSIÓN}

El estudio, nos ha permitido conocer la prevalencia de marcadores serológicos de la población atendida durante el periodo de estudio.

Los resultados pueden extrapolarse a otros bancos de sangre de la ciudad, dado que los donantes cumplen criterios de selección y calidad estandarizados por el PRONAHEBAS, lo que implica que los donantes comparten características básicas; aunque todos los bancos de sangre orientan sus procesos bajo normativas de aplicación nacional, lo que derivaría en su homogenización; la diversidad poblacional y las conductas de riesgo inconstantes dificulta su extrapolación a servicios de salud del país. 
Seroprevalencia de marcadores infecciosos causantes de pérdidas de hemodonaciones en el Servicio de Banco de Sangre del Hospital Nacional Docente Madre Niño San Bartolomé de enero 2008 a diciembre del 2013

Se tamizaron 11,399 unidades de sangre, con un aumento promedio de $15 \%$ de donaciones por año. Esto principalmente por donaciones voluntariarelacionadas, por reposición y depósito. Lo que demuestra el escaso nivel de donación voluntaria del país (30).

La OPS reportó prevalencia de $0,22 \%, 4,70 \%, 0,44 \%$, $1,11 \%$ y $0,46 \%$ para $\mathrm{VIH}$, VHB, VHC, sífilis y Chagas, respectivamente, en unidades de sangre tamizadas en Perú durante el 2011 (12).

Estos datos, son congruentes con lo hallado en el presente estudio (prevalencia global hallada del $9,36 \%)$, muy por encima de países de la región (40).

La prevalencia de hepatitis B se mantuvo constante (4.63\%), indicando una prevalencia importante de la infección parecida a la de otros países $(41,42)$.

Considerándose como factores de riesgo: ITS, trabaj os de alto riesgo, contacto sexual extramarital y juventud, igualmente, la vía horizontal como la más frecuente de trasmisión $(43,44)$. Otros factores son: bajo recurso económico, nivel educativo, hacinamiento y pobreza (45-48).

Resulta poco probable la transmisión de sífilis por transfusión (49). La tasa hallada fue de $1.78 \%$ por encima del último informe regional para el Perú (1.11\%). Es por ello, que el carácter subrogante de este marcador dentro del tamiz de banco de sangre posee un carácter relevante, aunque su asociación a otros marcadores de transmisión sea bajo, su cribado en algunos hemoderivados, principalmente plaquetas, es significativo en: Paraguay, Perú entre otros, siendo significativo en salud pública (29).

La prevalencia encontrada para HTLV (1.21\%) permanece sobre el porcentaje establecido por la OPS, para donantes peruanos. Popovic (50) señala que la evidencia serológica de anticuerpos anti-HTLV podría ser muy valiosa para identificar unidades de sangre o productos sanguíneos que puedan ser portadores potenciales del VIH. Otros estudios $(51,52)$, afirman que altos porcentajes de ese marcador depende del estado de la enfermedad y de la población de riesgo, en esta investigación, solo encontramos una asociación entre estos marcadores infecciosos.
La emigración y los problemas económicos, son considerados principales causas del tránsito de la enfermedad de Chagas hacia países no endémicos. El Perú, es un país endémico, por lo que; los Servicios de Banco de Sangre, deberían considerar que la gran parte de donantes son un potencial riesgo, que aumenta en relación con otros factores: estado inmunológico del receptor, la concentración del parásito y el tipo de cepa (53).

Se halló una prevalencia para Trypanosoma cruzi de $0.55 \%$ coincidiendo con la disminución de positividad para este marcador descrito por la OPS, sin embargo, la especificidad de las pruebas convencionales se ve afectada por falta de pruebas confirmatorias o falta de estandarización de los reactivos y por reacciones cruzadas con Leishmania spp y $\mathrm{T}$. rangeli, lo que ocasiona resultados indeterminados o borderline, ante esto, la transsialidasa posee alta sensibilidad y especificidad, suficiente como para ayudar al diagnóstico de resultados inconclusos (54-56).

Considerando que el VIH es el agente infeccioso con mayor impacto en salud pública, su escrutinio como potencial agente de contagio por vía transfusional es trascendental (57).

La prevalencia de $\mathrm{VIH}$ en los donantes de sangre ha disminuido de manera constante, $0.51 \%$ para el 2005-2010 a 0.41\% para el 2013, nuestros resultados muestran una tasa muy por debajo del promedio $(0.16 \%)$ debido a medidas eficaces que se tomaron para prevenir el $\mathrm{VIH}$, y mantenerse constante con reportes anteriores $(32,58)$.

La asociación entre marcadores para un solo donador, fue principalmente por ruta de contagio comunes (59). Los tres componentes que explican la varianza de todas las pruebas resultaron en: Primero, la relación entre HTLV I-II, VHB (HBsAg y $\mathrm{HBcAb}$ ) y Chagas, asociados por ser enfermedades crónicas y porque la infección está concentrada en determinados grupos poblacionales (60). Segundo, consideramos que la separación del VHC del acervo de componentes se debe especialmente al factor de contagio por exposición ocupacional, ya que comparte cronicidad y vías de transmisión parenteral y no parenteral con los demás marcadores infecciosos, aunque aún no estén esclarecidos (58). 
Por último, la relación subrogante entre VIH y Sífilis, reafirma la importancia del sinergismo entre ambos, por otra parte, hallamos una correlación negativa anómala para estos componentes (61).

Para el Perú, el descarte de unidades de sangre a causa de marcadores positivos aumentó de $0.82 \%$ el 2010 a 1.54\% el 2011 (40). La cantidad de sangre total eliminada durante nuestro periodo de estudio fue de $8.91 \%$ (1016 donaciones) con aumento progresivo en promedio de $16 \%$ por año, $2 \%$ más que el aumento de donaciones desde el 2008. Las 1016 unidades deshechadas trajeron como principal consecuencia la pérdida de sangre (457.2 Litros) la misma que ocasiono $172,720.00$ nuevos soles perdidos durante el periodo de estudio $(61,893.28$ USD). El costo total del estudio completo del donador en un Hospital privado vale 14\% y $65 \%$ más que en uno Institucional en México y USA, respectivamente, se desconocen aproximaciones análogas en el Perú (36).

El gran impacto económico por hemoderivados deshechados y la disminución del suministro de sangre por el banco de sangre, demuestran las limitaciones en la cadena de donación. Igualmente, la caducidad biológica, las alícuotas de paquetes globulares en neonatos y la donación autóloga, mínimamente beneficiosa y más costosa que la alogenica, contribuyen al problema (62).

La constancia de estos marcadores y la prevalencia de enfermedades en la comunidad se debe, ocasionalmente, a la poca información sobre su transmisibilidad y deficientes políticas de atención primaria y prevención de la salud, por consiguiente la concurrencia a la citación para emitir y dar a conocer resultados, como para el asesoramiento y el tratamiento; ya sea por el Centro de Salud como por el Centro para el Control y Prevención de Enfermedades de la localidad, es conveniente. Sin embargo, hoy en día, aún se observan dificultades por razones económicas, de distancia y conocimiento previo. Disminuir la seroprevalencia hallada en nuestros hemodonadores es un reto importante para mejorar la calidad de la transfusión, disminuir el costo económico y la incertidumbre por donador, y por tanto, asegurar la transfusión de sangre segura.
En conclusión, reducir el riesgo transfusional es un imperativo de los Servicios de Banco de Sangre y hemoterapia, para así administrar sangre segura.

La seroprevalencia investigada permanece elevada debido a una inconstancia geográfica de marcadores serológicos en hemodonadores del país, pobres políticas de salud y deficiencias en la cadena de donación.

Las tasas reportadas son coherentes con los reportes de la OPS y coinciden con otros estudios.

La selección de los donantes mediante entrevista o interrogatorio es insuficiente para el descarte o selección de hemodonaciones. Es importante confirmar casos reactivos con pruebas confirmatorias, usando tecnologías de tamiz dirigidas hacia la población en uso, con un eficiente sistema de control de calidad.

Las buenas prácticas en medicina transfusional, la implementación de nuevas metodologías de tamizaje y el desarrollo de estrategias para reducir las donaciones inseguras disminuirán la incertidumbre por hemodonación y asegurarán la transfusión de unidades seguras.

\section{Fuentes de financiamiento}

Autofinanciado por los autores.

\section{Conflictos de interés}

Los autores declaran no tener ningún conflicto de interés.

\section{Correspondencia:}

Moya Salazar, Jeel J

Dirección: Jr. Pacífico 957. Lima 07. Lima, Perú

Teléfono: 3652300

Correo electrónico: jeelms@outlook.com 
Seroprevalencia de marcadores infecciosos causantes de pérdidas de hemodonaciones en el Servicio de Banco de Sangre del Hospital Nacional Docente Madre Niño San Bartolomé de enero 2008 a diciembre del 2013

\section{REFERENCIAS BIBLIOGRÁFICAS}

1. Shimian Zou, et al. Donor Testing and Risk: Current Prevalence, Incidence, and Residual Risk of Transfusion-Transmissible Agents in US Allogeneic Donations. Transfusion Medicine Reviews. 2012; 26 (2): 119-128.

2. Gabriele WW. Incidence of bacterial transmission and transfusion reactions by blood components. DRK-Blutspendedienst West, Institut für Transfusions medizin Münster, Germany. Clinical Chemistry and Laboratory Medicine. 2008; 46(7):919-25.

3. Kuehnert MJ, et al. Transfusion-transmitted bacterial infection in the United States, 1998 through 2000. Division of Healthcare Quality Promotion, CDC, GA,USA. Transfusion. 2002; 41(12):14939.

4. Organización Panamericana de la Salud. Riesgo de transmisión de enfermedades infecciosas por transfusión de sangre en Centroamérica y Suramérica. Rev Panam Salud Publica 1998; 4(3).

5. Buitrago B. Patología geográfica, historia natural de las hepatitis B y D en Colombia. Biomédica, 1991; 11(4).

6. American Association of Blood Banks. Transfusion-transmitted disease. In: AABB Technical Manual. 14 Ed. Bethesda. 2002; 613. 23 .

7. Martin VC. Montoro AJ. Manual de Medicina Transfusional. SETS. 1994; 2:11-17.

8. Damian FR. El riesgo de transmisión de enfermedades infecciosas por vía de la transfusión. Ginecol Obstet Méx 1998; 66 (7): 277 283.

9. Choudhury N, Phadke S. Transfusion transmitted diseases. The Indian Journal of Pediatrics 2001; 68(10): 951-958.

10. Cortés BA, Gutiérrez MG. Prevalencia de marcadores para infecciones transmisibles por transfusión en donantes voluntarios. Colombia Med 1996; 27: 3-10.

11. Yan Hong, Xia Huang, Hua Ling, Hongwen Liao. Prevalence and trend of HIV infection among voluntary blood donors in China since implementation of the Blood Donation Law: a systematic review and meta-analysis. Tropical Medicine and International Health. 2012; 17(8):978-988.

12. Supply of Blood for transfusion in the Caribean and Latin American Countries 2010-2011. Washington, DC. Organización Panamericana de la Salud, 2013.

13. Scott DA, et al. A seroepidemiological survey of viral hepatitis in the Yemen Arab Republic, US Naval Medical Research Unit Number Three, Cairo, Egypt. Transactions of the Royal Society of Tropical Medicine and Hygiene. 1990; 84(2):288-91.

14. Paraná R, Almeida D. HBV epidemiology in Latin America. CastroHepatology Unit. J Clin Virology 2006; 34 (1): 130-133.

15. Lavanchy D. Hepatitis B virus epidemiology, disease burden, treatment, and current and emerging prevention and control measures. J Viral Hepatitis. 2004; 11(2):97-107.

16. Grassi DF et al. Prevalence and Factors Associated with Hepatitis B Virus Infection Among Senior Citizens in a Southern Brazilian City. Hepatitis Monthly. 2013; 13(5):7874.
17. Czernik G. Cuenca E, Dabski M. Seroprevalencia chagásica en hemodonantes del banco de sangre central de corrientes. Rev Posgrado VI Cátedra de Medicina. 2006: 160:05-08.

18. Schmuñis GA. Trypanosoma cruzi, the etiologic agent of Chagas disease: status in the blood supply in endemic and non-endemic countries. Transfusion. 1991; 31: 547-57.

19. Shimian Z, Stramer SL, Dodd RY. Donor Testing and Risk: Current Prevalence, Incidence, and Residual Risk of TransfusionTransmissible Agents in US Allogeneic Donations. Transf Med Rev 2012; 26(2):119-128.

20. Alleyne GA. El próximo cuadrienio. Washington, DC. Organización Panamericana de la Salud; 1998.

21. Smith D, Dood R. Transfusion-transmitted infections. Chicago. ASCP Press. 1991.

22. Center for Biologics Evaluation and Research. Revised Precautionary Measures to Reduce the Possible Risk of Transmission of Creutzfeldt-Jakob. Disease (CJD) and New Variant CreutzfeldtJakob Disease (nvCJD) by Blood and Blood Products. Guidance for Industry.U.S. 1999; 03-07.

23. Emond RT, Evans B, Bowen ET, Lloyd G. A case of Ebola virus infection. Br Med J. 1977; 27(2):541-544.

24. Tambyah PA, et al. Dengue Hemorrhagic Fever Transmitted by Blood Transfusion. N Engl J Med 2008; 359:1526-1527.

25. Will R, Kimberlin R. Creutzfeldt-Jakob disease and the risk from blood or blood products. Vox Sang 1998; 75:178-80.

26. Bryan S, et al. Risk for malaria in United States donors deferred for travel to malaria-endemic areas. Transfusion. 2009; 49(11): 2335-2345.

27. Blejer J, Saguier M, Salamone H, Carreras VL. Seroprevalencia de citomegalovirus en donantes de sangre. Hemoterapia 1995; 7: 149.

28. Mooltes D. Transfusion transmitted Cytomegalovirus. Pediatr Pathol Mol Med 2000; 19: 149-59.

29. Blejer LJ, y col. Riesgo de Transmisión de Infecciones por vía Transfusional. Medicina. 2002; 62:259-278.

30. World Health Organization. Percentaje of voluntary unpaid blood donations. 2007.

31. Mazzur S, y col. Distribución de marcadores serológicos del virus de la hepatitis $B$ en la sangre de donantes de 13 países del hemisferio occidental. Actas del Taller Latinoamericano de la Cruz Roja sobre Hepatitis B. Bol Of Sanit Panam 1980; 89:239-47.

32. Yuen, A. Transmisión sanguínea del VIH en el Perú. Seminario de SIDA. Comisión Nacional de SIDA.MINSA. Lima- Perú. 1988.

33. De Schyver A, Meheus A. Syphilis and blood transfusion: A global perspective. Transfusion 1990;30:844-847.

34. Lubarki AD, Hahn C. The hospital cost (Fiscal year 1991-1992) of a simple perioperative allogenic Red Blood Cell Transfusion During Elective Surgery at Duke University. Anesth Analg. 1994:74:236-42.

35. Roche JK, Stenglo JM. Open heart surgery and the demand for blood. JAMA. 1988;225:1516-21. 
36. Sanchez AS, Gonzales NP, Alvarez VJ. Costos en la Transfusión Sanguínea. Rev Mex Anest 2000;23:66-70.

37. Vogler $\mathbf{I H}$, et al. Effectiveness of confidential unit exclusion in screening blood donors of the regional blood bank in Londrina, Paraná State. Rev brasileira de hematologia e hemoterapia.2011; 33(5):347-52.

38. Locke SE, et al. Computer-based interview for screening blood donors for risk of HIV transmission. Center for Clinical Computing, Boston. JAMA. J Am Med Association. 1992; 268(10):1301-1305.

39. Ownby et al. Loss of volunteer blood donors because of unconfirmed enzyme immunoassay screening results. Retrovirus Epidemiology Donor Study. Transfusion. 1997 ;37(2):199-205.

40. De La Cruz SR, Barrera CT, Vidal EJ, Rodríguez SI. Marcadores serológicos de sífilis, hepatitis $\mathrm{B}$ y VIH en donantes de sangre en el Hospital Nacional Cayetano Heredia, Lima-Perú. Rev Med Hered 1999; 10(4).

41. Oliveira C, et al. Seroprevalence of hepatitis B virus infection in individuals with clinical evidence of hepatitis in Goiânia, Goiás. Detection of viral DNA and determination of subtypes. Revista do Instituto de Medicina Tropical de São Paulo. 2002; 44(6):331-4.

42. Ramírez SM, Huichi AM, Aguilar EG, Pezo OJ. Seroprevalencia de hepatitis viral $B$ en estudiantes universitarios en Abancay, Perú. Rev Perú Med Exp Salud Publica.2011;28(3):513-7.

43. Tahereh V, Moayed AS, Ali K, Jafar K, Babak Y. Hepatitis B Prevalence and Risk Factors in Blood Donors in Ghazvin, Iran. Hepatitis Monthly 2005;3.

44. Sellors J, Vincetic MZ, Howard M, Mahony JB, Chernesky MA. Predictors of positivity for hepatitis $\mathrm{B}$ and the derivation of a selective screening rule in a Canadian sexually transmitted disease clinic. J Clin Virology. 1998; 11(1):85-91.

45. Nuchprayoon T, Chumnijarakij T. Risk factors for hepatitis B carrier status among blood donors of the National Blood Center, Thai Red Cross Society. The Southeast Asian journal of tropical medicine and public health. 1992; 23(2):246-53

46. Ahizechukwu CE, Uzoamaka AE, Charles IO, Ifeanyichukwu UE, Chukwuanugo O. Prevalence, correlates and pattern of hepatitis B surface antigen in a low resource setting. Virology Journal 2011; $8: 12$.

47. Ruiz CR, y col. Marcadores serológicos de hepatitis viral en personal hospitalario. Estudio comparativo con una población control. Diagnóstico. 1985; 16:142-145.

48. Azimi PH, et al. Transfusion-acquired hepatitis $\mathrm{A}$ in a premature infant with secondary nosocomial spread in an intensive care nursery. Am J Dis Child 1986; 140: 23-7.

49. Tramont E. Treponema pallidum (syphilis). In: Mandell G, bennett $\mathrm{J}$, Dolin R. Principles and practice of the infectious diseases. $4^{\circ}$ ed. New York. Churchill Livingstone. 1995:2117-33.

50. Popovic M. Detection, isolation and continuous production of cytopathic retroviruses (HTLV-III) from patientswith AIDS and PreAIDS. Science 1984;224:497-500.

51. Gotuzzo E, et al. The impact of human T-Lymphotrophic virus type I/II. Infection on the prognosis of sexually acquired immunodeficiency syndrome. Arch Intern Med 1992; 152.
52. Sarngadhasan MG. Antibodies reactive with human T-limphotropic retro viruses (HTLV-II) in the serum of patients with AIDS. Science 1984;224: 506-8.

53. Nickerson P, Pamela O, Schroeder M, Sekla L, Johnston J. Transfusion-associated Trypanosoma cruzi infection in a nonendemic area. Ann Intern Med 1989; 111: 851-3.

54. Blejer JL, Sartor PA, Bottasso, O, Salamone HJ, Leguizamón, MS Trans-sialidase Inhibition Assay for the detection of Trypanosoma cruzi infection in blood donor samples from Argentina. Vox Sang 2008; 95: 189-196.

55. Assayag $M$, et al. Prevalencia de serología Positiva Hepatitis $C$ en Donantes de Sangre del Hospital Edgardo Rebagliati Martins de ESSALUD mayo-noviembre de 1998. Rev Asmeghor.1998; 3(2): 9-12.

56. Farfán G; Cabezas C. Prevalencia de la Hepatitis Viral C en donantes de sangre del Perú. Rev. gastroenterol. Perú 2003; 23(3): 171-176.

57. Busch MP, et al. Very low level viremia in HCV infectious unit missed by NAT. Transfusion 2003;43(8):1173-1174.

58. Busch MP, et al. Nucleic acid amplification testing of blood donors for transfusion-transmitted infectious diseases: report of the interorganizational task force on nucleic acid amplification testing of blood donors. Transfusion 2000; 40(2):143-159.

59. ONUSIDA. AIDS epidemic update 2009. November 2009.2009 Date. Available from: [URL] http.//data.unaids.org/pub/Report/2009/ JC1700_Epi_pdate_2009_en.pdf. Access: Feb 25, 2014.

60. Sánchez J, et al. Prevalence of sexually transmitted diseases and retroviral infections in relation to sexual behavior knowledge, attitudes and pactices among the general population in Lima, Perú. VIII International conference on AIDS, Amsterdam, NED.1992.

61. Funnyé $A S, A k h t a r A J$, Ven $D$. Syphilis and human immunodeficiency virus co-infection. J Natl Med Assoc 2003; 95(5):363-82.

62. Etchason J, et al. The cost effectiveness of preoperative autologous blood donations. N Engl J Med. 1995; 16(11):719-24. 